\title{
Inserción Laboral de Nuevos Investigadores con Grado de Doctor en Chile
}

\author{
Occupational Insertion of New PhD Researchers in Chile
}

\author{
Horacio Gonzalez', Alejandro Jiménez²
}

\begin{abstract}
The formation of new highly trained human resources is considered a key factor for social, cultural and economic development. During the last years, Chile has made a significant effort to increase the amount of Ph.D researchers. Nevertheless, there is no evidence from the National Innovation System (NIS) regarding the insertion capacity for this duplicated population of new researchers in the local labor market. Under such point of view, public investment is eventually under risk, due to the sub-optimal conditions to absorb and employ these professionals in activities related to science research and technological development in Chile.
\end{abstract}

Keywords: highly trained human resources; postdoctoral scholars; scientific knowledge; technological development; Chile; public policy.

La formación de nuevo capital humano avanzado es clave para el desarrollo social, cultural y económico de las naciones. En los últimos años, Chile ha realizado un esfuerzo considerable por aumentar la cantidad de investigadores con grado de doctorado. Sin embargo, no hay evidencia que el Sistema Nacional de Innovación (SNI) chileno tenga la capacidad para insertar a estos nuevos investigadores que vendrían a duplicar la población de científicos en Chile. De esta forma, la inversión pública en esta materia corre el eventual riesgo de que los nuevos doctores no encuentren las condiciones para insertarse laboralmente en funciones profesionales propias de investigación científica y desarrollo tecnológico dentro de Chile.

Keywords: capital humano avanzado; becas postdoctorales; conocimiento científico; desarrollo tecnológico; Chile; política pública.

\footnotetext{
'Investigador independiente. E-mail: dhzlatar@gmail.com

${ }^{2}$ Facultad de Economía y Negocios, Universidad Alberto Hurtado. Erasmo Escala 1835 - Santiago, Chile. E-mail: ljimenez@uahurtado.cl
}

ISSN: 07 I8-2724. (http://www.jotmi.org) 


\section{I.- Introducción}

A partir de 2014 y hasta el 2018, según cifras preliminares, más de cuatro mil nuevos investigadores chilenos obtendrán el grado académico de doctor (ver gráfico I), luego de cumplir sus estudios de postgrados tanto dentro como fuera del territorio nacional. Lo anterior, de acuerdo a estimaciones posibles de realizar en base al documento " 25 Años Becas de Doctorado CONICYT", publicado a comienzos del 2014 por el Programa de Formación de Capital Humano Avanzado de CONICYT. En este informe se indica que hay más de 6.200 candidatos a doctor confirmados entre el año 2005 y el 2012.

Si consideramos que actualmente existen en Chile 4.559 investigadores categoría Fondecyt ${ }^{3}$ (Conicyt, 2013a), podemos proyectar que habrá un fuerte aumento del número de investigadores generando una inusitada oferta de capital humano avanzado y disponible para ingresar al sistema académico y/o productivo en Chile.

El presente artículo tiene por objetivo principal levantar información referente a las tres principales vías de inserción que tienen a disposición los investigadores: academia, industria y emprendimientos propios. También se presentan algunos indicadores y prácticas de inserción de capital humano avanzado mediante la implementación de políticas públicas en Chile.

\section{I.I.-Aumento de investigadores con grado de doctor en Chile, formados con financiamiento público, y su inserción laboral}

El 23 de Octubre del 2008, el Gobierno de Chile creó el Programa Becas Chile, como respuesta a la necesidad y urgencia de contar con académicos, profesionales y técnicos de excelencia, que adquieran las competencias para el desarrollo científico y tecnológico, la innovación y el emprendimiento. Aquella iniciativa se enmarcó dentro del plan de acción gubernamental enfocado a la educación e innovación, cuyo fin primordial es insertar a Chile en la sociedad del conocimiento y acelerar el camino hacia el desarrollo económico, social y cultural. Una manera concreta de actuar en esta línea, es fortaleciendo las capacidades de investigación científica básica y aplicada, el desarrollo tecnológico, la innovación y el emprendimiento (Mineduc, 20I0).

${ }^{3}$ El Fondo Nacional de Desarrollo Científico y Tecnológico (Fondecyt) es un programa público administrado por Conicyt, destinado a estimular y promover el desarrollo de la investigación científica básica en Chile a través de fondos concursables. Por investigadores Fondecyt se entiende como investigadores responsables o co-investigadores de proyectos Fondecyt Regular e Iniciación entre 2006 y 201 I, contados por RUT distintos y únicos (Conicyt, 20I2).
Durante el cuatrienio 2008-20I2, 2.44I postulantes fueron seleccionados para realizar estudios de doctorado fuera de Chile (Mineduc, 2014). Mientras que en el mismo período, se reportó que 2.164 estudiantes iniciaron efectivamente sus estudios de doctorado en el extranjero; a éstos se suman 2.639 becarios quienes también comenzaron los mismos estudios en territorio nacional durante los mencionados cuatro años (Conicyt, 20I4a). En total, suman 4.803 beneficiarios que iniciaron sus estudios entre los años 2008 y 20 I2. Si consideramos que hoy en día existen 4.559 investigadores categoría Fondecyt en Chile (Conicyt, 2013a), cifra comparable a los 4.741 investigadores con grado de Doctor que reportó el Ministerio de Economía (Minecon, 20I4), se doblaría el número de científicos, produciendo una inusitada oferta de capital humano avanzado disponible a ingresar al sistema académico y productivo en Chile.

Estimaciones de este artículo, realizadas exclusivamente en base a Conicyt (2014a), indican la posibilidad de que a partir del 2014 y hasta 2018 , se podrían titular anualmente en promedio más de 800 nuevos investigadores con el grado de doctor (ver gráfico I).

Gráfico 1: Distribución de la estimación del número de doctores titulados con beca Conicyt

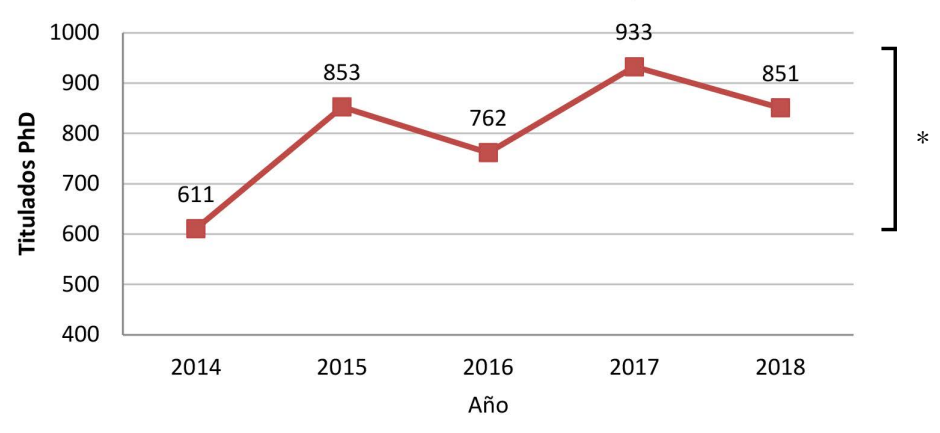

* [40I0 nuevos doctores estimados para el 20I8] Fuente: Elaboración propia en base a Conicyt (2014a)

ISSN: 07 I8-2724. (http://www.jotmi.org) 
De esta forma, si sumamos los 4.010 doctores estimados a los actuales 4.559 investigadores categoría Fondecyt (Conicyt, 2013a) podemos proyectar un total de 8.568 investigadores para el 2018. Lo anterior representaría un crecimiento para el periodo de un $88 \%$, según se observa en el gráfico 2 . La mencionada proyección está subestimada, pues considera exclusivamente a doctores que estudiaron con beca CONICYT y no a aquellos que utilizaron otras fuentes de financiamiento. Lo anterior implica que el crecimiento real proyectado podría llegar a ser incluso más alto que el indicado por el gráfico 2 (ver consideraciones metodológicas).

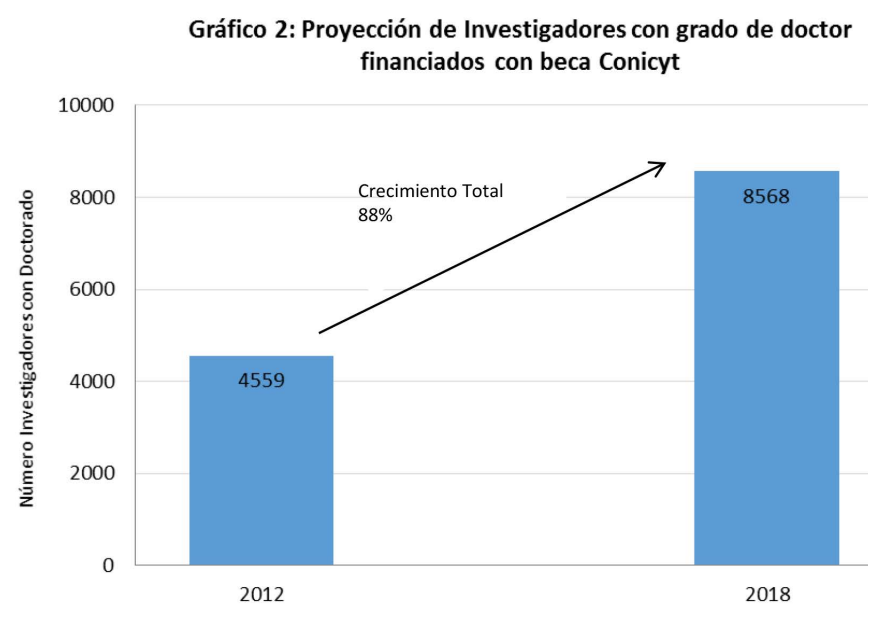

Fuente: Elaboración propia en base a Conicyt (20I4a)

Es importante advertir, que a pesar de que el aumento del capital humano avanzado, disponible para la investigación es fundamental para el desarrollo del país, no hay evidencia que el Sistema Nacional de Innovación (SNI) sea capaz de albergar esta nueva cantidad de investigadores con grado de doctor. La principal razón es la carencia de una política pública concreta que comprometa y defina el rol que tendrán los nuevos investigadores en el SNI (más adelante discutiremos con mayor profundidad las políticas públicas que el país tiene en materia de inserción de capital humano avanzado). La segunda razón es que en Chile, las universidades dependen casi exclusivamente de fondos estatales para financiar su investigación científica y tecnológica. Desafortunadamente, el presupuesto público del componente de Ciencia, Tecnología e Innovación (CTI) ha desacelerado marcadamente su crecimiento desde el año 2010 , lo que ha significado una caída de la inversión como porcentaje del PIB alcanzando un promedio del $0,35 \%$. Este valor palidece frente al 2,4\% de la OECD (Minecon, 20I4); organización de la cual Chile es miembro desde el 2010.
A pesar de lo anterior, en los últimos años, se han hecho esfuerzos focalizados destinados a mejorar las posibilidades de inserción de los nuevos investigadores. Por ejemplo, en 2009, Conicyt creó el Programa de Atracción e Inserción (PAI); además, esta misma institución aumentó en $50 \%$ los recursos disponibles para Fondecyt desde 2010 (concretamente el presupuesto para el instrumento de Postdoctorado Nacional creció $40 \%$ desde 201 I); mientras que en 2012, se formó el Programa de Equipamiento Científico y Tecnológico, Fondequip (Aguilera, 2012). Éstos, entre otros esfuerzos, se enmarcan en el crecimiento del presupuesto de Conicyt en 2,6 veces desde U\$ 207 millones de dólares, en el año 2008, a U\$ 546 millones de dólares, en 2013 (Conicyt, 20I3a), el cual busca entregar condiciones superiores para el desarrollo científico y las siguientes generaciones de investigadores.

Sin embargo, estas medidas todavía parecen ser insuficientes y no resuelven el problema de fondo de la inserción de doctorados en el país. Miembros de la comunidad científica nacional y organizaciones internacionales han criticado la falta de una política de Gobierno moderna para la investigación en Chile (OECD y Banco Mundial, 2009; Feller, 20I I). A modo de ejemplo, aseguran que el Plan Nacional para la Ciencia y el Desarrollo no se ha modificado desde 1988, figurando entre los más antiguos de la región (Astudillo et al, 2012). Además, claman por la falta de una política de Estado más profunda, que impulse un plan de largo plazo para la ciencia, la que a su juicio se podría resolver con la formación de un Ministerio de Ciencia y Tecnología (Catanzaro, 20I4).

Bajo este panorama, Pérez (20/2) fundamenta que la inserción de doctores en la academia y la industria constituye un tema preocupante, pues a la actual tasa de generación de doctores, en un par de años más existirán miles de investigadores intentando insertarse en el mundo académico, donde las universidades ofrecen escasos puestos laborales de investigación; o bien, buscando trabajo en el esquivo mundo productivo, donde las cifras muestran tasas de inserción laborales aún menores que en la academia (ver gráfico 3).

En síntesis, la inserción laboral de los nuevos titulados de doctorado es un elemento que seguramente estará presente en la agenda de la política pública en ciencia, tecnología e innovación durante los próximos años, en Chile. 


\section{I.2.- Inserción Laboral de Investigadores}

La inserción laboral es entendida como el proceso en el cual una persona ingresa a un puesto de trabajo estable, luego de un tiempo donde estuvo dedicada a otras actividades no remuneradas, principalmente obteniendo formación profesional y técnica en la educación superior, en la convicción de que estas actividades los habilitará para una mejor inserción en el mercado del trabajo (Rodríguez, 2004). En la mayoría de los casos, la inserción laboral está caracterizada por una competencia desmedida, donde una gran oferta de capital humano postula a una demanda relativamente reducida. Si esta situación ya convierte a la inserción en algo complejo, en algunos casos se suma la necesidad económica del individuo por obtener una fuente de ingreso permanente. De esa forma, se desprende que la inserción es un momento crítico en la vida de cada persona, en la cual existen pocas certezas (Martínez, 1998).

En el caso de los investigadores recién titulados de su doctorado, el mercado laboral también es estrecho $y$, a primera vista, la carrera de investigación es poco atractiva en términos salariales, considerando el alto nivel de especialización que se requiere (Kidd y Green, 2006).

Así, en EE.UU., un estudiante de ciencia o ingeniería que optó por una carrera en el sector financiero obtiene un sueldo de entrada cinco veces superior al de un colega que eligió la investigación y cuyo primer trabajo es como ayudante de laboratorio (Gaglani, 2009). Estas diferencias salariales se acentúan cuando el $\mathrm{PhD}$ accede a una posición postdoctoral (Stephan, 2012). Más allá de las consideraciones económicas, el objetivo declarado de los estudiantes por continuar una carrera en ciencias es "el amor por el conocimiento, no los salarios y trabajos" (Freeman, 1989). Es por esto, que la mayoría de los graduados de doctorado manifiestan una fuerte preferencia por insertarse laboralmente en la investigación académica (Davis, 2005; Fox y Stephan, 200I). En Chile, esta situación también es similar, donde más del $80 \%$ del total de investigadores realiza labores en la academia, según gráfico 3 (Ricyt, 20I I; y Minecon, 20l4).

En general, los principales obstáculos que impiden la plena inserción de nuevos doctorados son la escasa demanda para el tamaño de la oferta laboral, la cual es aún más fuerte en el mundo académico debido a los bajos patrones de movilidad que presenta (Stephan, 2012). A esto se agrega la significativa inversión en términos de infraestructura, equipamiento y materiales en los cuales debe incurrir la organización contratante para entregar adecuadas condiciones a la investigación.
Si observamos la literatura y la experiencia internacional podemos identificar tres vías para la inserción laboral de investigadores con grado de doctor: postdoctorado, investigación en la industria y el emprendimiento propio.

\section{2.- Principales vías para la inserción laboral de doctores}

\section{1.- Postdoctorado}

El doctorado es el máximo grado académico al cual un estudiante puede optar. Las principales habilidades que consigue un doctorado en estos estudios es la creación de un conocimiento nuevo a través de la investigación. Esencialmente, es la educación y entrenamiento de los futuros científicos; período al que le sigue la posición postdoctoral (Neumann, 20I0).

El postdoctorado es una posición temporaria para quien ha completado recientemente sus estudios de doctorado (Auriol, 2010). Su objetivo es profundizar la experiencia de investigación en líneas de trabajo previamente abordadas por el doctorado, que así le permita adquirir nuevas capacidades y métodos. Desde que las primeras posiciones postdoctorales fueron reclutadas y entregadas por la Universidad Johns Hopkins (EE.UU.) en 1876, este instrumento se ha transformado en una parte integral de la investigación, así como en un elemento crucial para que los doctores prosigan sus carreras, especialmente en la academia (Stephan, 2006). No obstante, las posiciones postdoctorales eran una rareza durante la primera mitad del siglo XX; solo fue durante la décadas de 1980 y 1990 que su número creció significativamente, logrando suplir la falta de posiciones académicas que no podían hacer frente al aumento de titulación de nuevos doctorados (Davis, 2005). Al mismo tiempo, este tipo de beca fue responsable del $43 \%$ de la productividad científica conducida en EE.UU. (Vogel, 1999).

Culminado el postdoctorado se obtiene la calidad de "investigador independiente", estatus fundamental para alcanzar una posición laboral permanente en la academia y que además le permita postular a fondos para realizar nueva investigación, que por lo general se adjudican en torno a criterios de excelencia y experiencia. Sin esa condición de prestigio, sería muy difícil para los científicos lograr financiamiento público y proseguir sus líneas de I+D (Stephan, 2012). 
El nivel de competencia para ingresar a un postdoctorado es significativo. Por ejemplo, en Finlandia se estima que por cada tres nuevos graduados de PhD existe solo una posición postdoctoral disponible, mientras que en Israel la relación es I a 5 (OECD, 20I I). Asimismo, el becado de postdoctorado está bajo constante presión por publicar y levantar fondos, por lo que tiene escaso tiempo para desarrollar otras habilidades no relacionadas a la investigación y capaces de potenciar su competitividad laboral en otras áreas del conocimiento.

No obstante, $y$ a pesar de que el $\mathrm{PhD}$ puede tener una alta especialización, sus capacidades son de creciente interés en la industria, ya que así puede enfrentar los desafíos de la economía del conocimiento. En este contexto, las empresas privadas están dispuestas a contratar esas habilidades abriendo nuevas formas de inserción para los graduados de doctorado, incluso aquéllos que no cuentan con el postdoctorado (Gura, 20I2).

\subsection{Investigación en la Industria.}

El interés de la industria por atraer científicos con grado de doctor ha sido recopilado y analizado por una serie de autores. Entre los esperados efectos positivos que los $\mathrm{PhD}$ aportan a las compañías privadas se encuentran los conocimientos de punta en ciencia y tecnología (Rosenberg, 1985; Brooks, 1994; Cockburn y Henderson, 1998; Cohen et al., 2002; Stephan, 2006). A través del proceso industrial aplicado, este conocimiento es capaz de convertirse en activos de propiedad intelectual que otorgan ventajas a la empresa (Sauermann y Cohen, 2008). Además, la inserción del PhD en la industria empuja a la conformación de redes con el resto de la comunidad científica (Sauermann y Stephan, 2009). De esa forma, los doctores otorgan canales de conocimiento crítico para las empresas constituyéndose muchas veces en el pilar de su competitividad (Roach, 2009).

En países donde la $I+D$ tiene una alta importancia en el quehacer productivo, la industria ofrece sueldos más altos a cientíicos e ingenieros lo que favorece la empleabilidad en la industria (Sauermann y Stephan, 2009). Incluso, algunos autores plantean que las universidades podrían estar perdiendo su atractivo laboral debido a la excesiva dependencia de fondos públicos para desarrollar investigación, donde las agencias de financiamiento estatal están crecientemente apoyando "ciertas temáticas de investigación" en desmedro de otras áreas del conocimiento (Roach y Sauermann, 20I0). Esto último es visto como una contracción a la libertad académica debido al continuo deseo de la política pública por comercializar la investigación en sus distintas formas de transferencia tecnológica, generando un debate en torno al rol de la ciencia como un bien público. En EE.UU. aquello ha producido que en casos puntuales un investigador deba atrasar su publicación por consideraciones comerciales (Murray y Stern, 2007). Algunos autores señalan que las tradicionales normas de la academia en ese sentido se han deteriorado, además de la progresiva desaparición de salarios "garantizados", lo cual ha llevado a los titulados de doctorado a plantearse un puesto laboral en el sector productivo como opción profesional.

La investigación en la industria ha convergido en interesantes modelos de asociación, generando lazos donde incluso existen incentivos de la propia empresa para concretarlos (Roach y Sauermann, 2010). Sin embargo, y a pesar de los beneficios que los investigadores aportan a la industria, la relación entre ambos no es fluida. En Chile, esta situación es crítica y son pocas las empresas que contratan personal especializado para realizar actividades de I+D.

De acuerdo a la 3era Encuesta de Gasto en I+D (Minecon, 2014), las empresas chilenas emplearon durante 2012, en total, a 185 investigadores con grado de doctor. Finlandia, que posee un tercio de la población total de Chile, tiene más de 23 mil doctores en la industria (OECD, 20II). En Chile, la mayoría de los investigadores tiene un "gusto por la ciencia", que los motiva a estar más cerca de la academia que de la industria. Las universidades son observadas por los investigadores como un ambiente con altos niveles de libertad, recursos y seguridad laboral (Roach y Sauermann, 2010), donde la principal recompensa es el reconocimiento que hacen sus pares en cuanto a los descubrimientos realizados (Sorenson y Fleming, 2004).

\section{3.- Emprendimiento}

La tercera vía bajo la cual un científico puede optar a su inserción laboral es en llevar personalmente los resultados de su investigación al mercado a través de la creación de una empresa (emprendimiento). El concepto de emprendimiento con base científica consiste en la dedicación simultánea que realiza el investigador en cuanto a sus actividades académicas y la explotación económica (Lehrer y Asakawa, 2004). Esta acción tiene la lógica de facilitar la transferencia tecnológica y desarrollar innovación hacia la industria, con la participación comercial de miembros académicos (Mowery y Sampat, 2004; Geuna y Nesta, 2006). Las políticas públicas han puesto incentivos para que la investigación se enfoque en proteger la propiedad intelectual, para luego obtener beneficios económicos en la comercialización de la misma (Gulbrandsen y Etzkowitz, 1999; Powel et al, 2007). El caso de Estados Unidos es el más significativo, luego de la promulgación del Acta Bayh-Dole en 1980, que permitió a las universidades proteger y registrar como propios los resultados de sus investigaciones financiadas con fondos federales (Stevens, 2004). 
El emprendimiento científico requiere de varias condiciones para su proliferación, como involucramiento previo del científico con la industria, productividad científica del investigador, acceso al capital de riesgo y potenciales demandantes de tecnología (Haessler y Colyvas, 2010). Al mismo tiempo, los riesgos e incertidumbres son significantes en la transferencia y comercialización de la I+D (Foray y Lissoni, 2010), lo cual incluso ha motivado a instituciones de gobierno a realizar entrenamiento a los involucrados para realizar emprendimientos de base científica (Ojala y Heikkila, 20I I).

De esta forma, el proceso de generar y capturar información para conformar un emprendimiento, involucra a varios participantes, quienes motivados por la resolución de problemas, incentivos de mercado, misiones institucionales, se encuentran con numerosos conflictos de coordinación; por tanto la existencia de fallas de mercado desalienta los esfuerzos por promover la innovación y hace compleja la cooperación en un proyecto de I+D (Benavente y Price, 20I4).

Chile no está exento de estos problemas. Una de las razones es la poca presencia del emprendimiento científico en la agenda de los investigadores chilenos, quienes no tienen como objetivo primordial atender las necesidades del sistema tecnológico de innovación a través del conocimiento aplicado (Bernasconi, 2005). Por otro lado, la inexistencia de capital de riesgo en el sistema financiero chileno para emprendimiento científicos es otra barrera muy difícil de franquear (Chandra y Medrano, 2012;Amorós et al, 2008). Además, el bajo interés de la industria nacional por conectarse con la investigación no permite generar condiciones de mercado que demanden este tipo de servicios (Benavente, 2006).
En los últimos años, el Gobierno de Chile ha tratado de revertir esa situación en las universidades, lugar donde se genera el conocimiento y la ciencia, ubicando incentivos que aumenten la innovación basada en la producción científica, a través de financiamiento institucional para la innovación en la educación superior (Mineduc, 20I3). Conicyt a través del Fondo de Fomento al Desarrollo Científico y Tecnológico (Fondef, creado en 1991) también ha lanzado concursos destinados a valorizar la investigación realizada en las universidades, buscando generar emprendimientos de base científica. No obstante, los resultados han sido exiguos debido a que en Chile, no existe una institucionalidad dentro de las universidades que permita transferir los resultados de la investigación básica a productos comerciales. Con mucho trabajo, algunas universidades chilenas han logrado avanzar en la implementación de políticas de propiedad intelectual dentro de sus planteles. Sin embargo, ha sido muchas veces la improvisación la encargada de resolver los problemas que surgen de la apropiación del conocimiento científico (Jiménez, 20II).

En la academia chilena, es normal la formalización de una sociedad empresarial al alero de disciplinas como la ingeniería o la administración, pero no en las áreas vinculadas a las ciencias básicas, como la biología, química y/o ciencias de la vida, todas ellas, disciplinas con eminente potencial innovador.

Incluso hay elementos en la propia ley chilena como el Estatuto Administrativo o la Ley de Probidad, que regula el régimen del Funcionario Público, y que podrían impedir que los académicos de universidades estatales realicen actividades emprendedoras dentro de la Universidad sobre ciertos montos bastante bajos para este ámbito (200 UTM). Además, las direcciones jurídicas, con respaldo de la Contraloría General de la República, han establecido un marco estricto y algo desmotivante para las actuaciones de académicos que destinan tiempo en actividades como los spin offs. Incluso podría representar un conflicto de intereses por la incompatibilidad legal que implica desarrollar actividades propias de un funcionario público por una parte y otras que buscan apropiarse y/o explotar comercialmente activos que surgen de un financiamiento estatal. Lo anterior se acentúa y se complejiza en un entorno de rechazo al lucro en la educación. (Jorge Rojas, Abogado, Doctor en Derecho de la Universidad de California, Berkeley, Comunicación Personal). 


\section{3.- El contexto en Chile}

De acuerdo a las políticas de inserción descritas con anterioridad se puede afirmar que los investigadores tienen tres caminos para continuar su carrera profesional: la academia, la industria; y emprendimientos propios. Sin embargo, y dado el escaso número de empresas de base científica y tecnológica creadas en Chile (Amorós y Echecopar, 2008), nos centraremos en las políticas públicas de inserción en la académica y la industria.

\section{I.- Inserción en la academia}

En Chile existe un total de 10.385 investigadores en I+D (indistintamente de su nivel educacional), según "Resultados 3era Encuesta Nacional sobre Gasto y Personal en Investigación y Desarrollo I+D 201 I-I2"; de ellos, 4.74I tienen el grado de doctor, lo que representa el $45,6 \%$ del total de investigadores (Minecon, 20/4). De acuerdo a la misma base de información, su principal fuente laboral es la educación superior con $80,4 \%$; mientras que el porcentaje de investigadores en empresas llega al 6,8\% (ver gráfico 3).

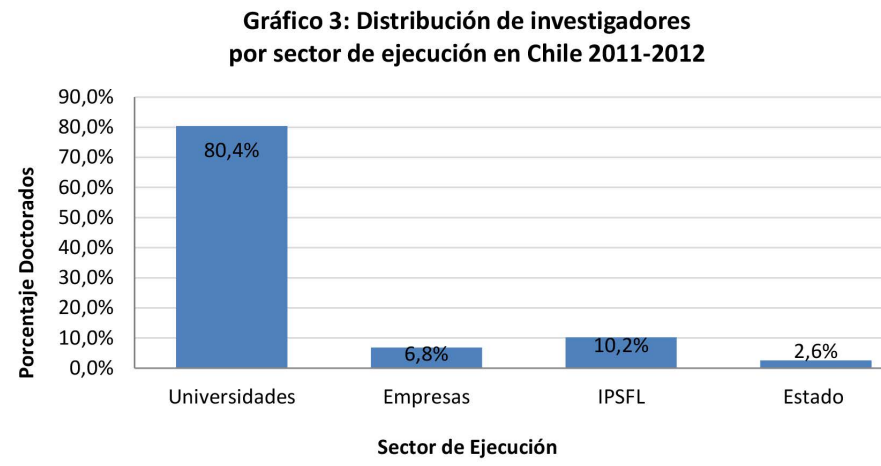

Fuente: Minecon (2014)

Nota: IPSFL corresponde a Instituciones Privadas sin Fines de Lucro
Conicyt es la principal agencia de apoyo y desarrollo de la investigación científica en Chile, con un presupuesto anual que bordea los U\$ 550 millones de dólares, y entrega un número importante de becas de postdoctorado para cursar especialización tanto dentro como fuera del territorio nacional. En sus bases de concursos, los Postdoctorados ofrecidos por Conicyt están dirigidos a investigadores que hayan obtenido su título de doctor dentro de los últimos tres años, tanto nacionales como extranjeros (en este último caso con residencia definitiva en Chile o bien visa temporaria). Durante los últimos cinco concursos, el instrumento de Postdoctorado Nacional ha beneficiado en promedio a 173 investigadores por año, lo que representa una alza de 4,3 veces, respecto a los 40 adjudicados en el período 2001-2008, según se observa en el gráfico 4 (Conicyt, 20l4b).

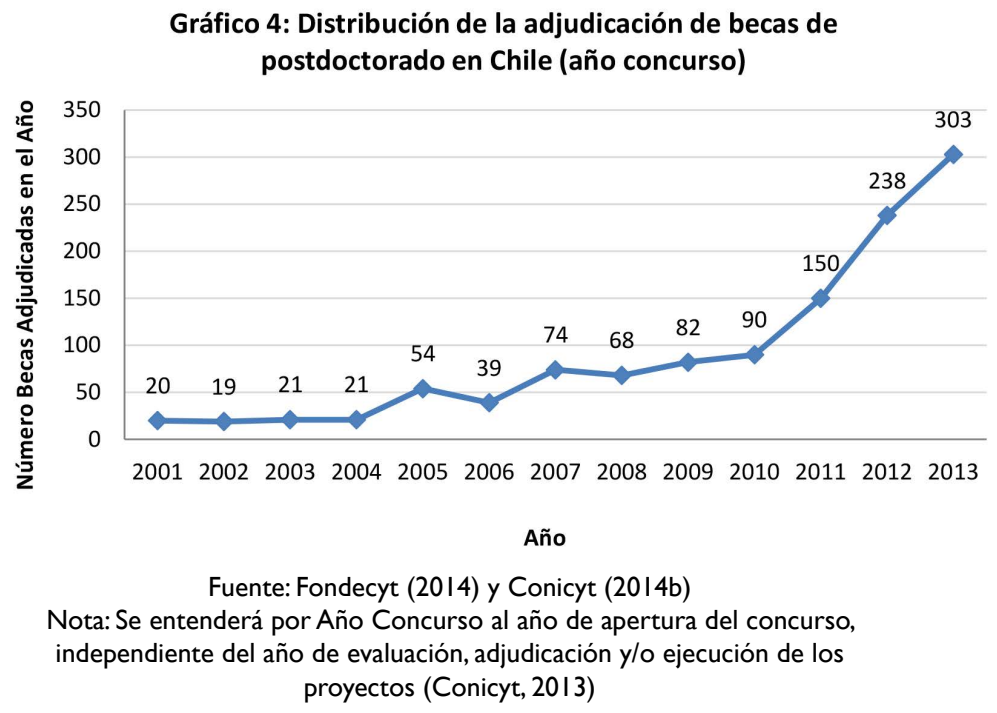

ISSN: 07 I8-2724. (http://www.jotmi.org) 
Por otra parte, el instrumento de Postdoctorado en el Extranjero que entrega el Programa Becas Chile está dirigido a investigadores nacionales y extranjeros (en este último caso con residencia definitiva en Chile), para realizar investigación en el extranjero, en proyectos de duración mínima por 6 meses y máxima por 24 meses. Durante el período 2008-2013, se otorgaron en promedio 42 becas por año para efectuar postdoctorados fuera del país (ver gráfico 5).

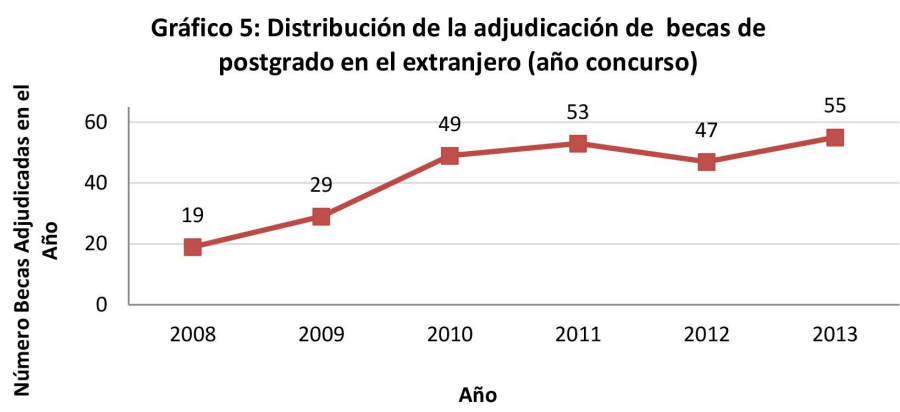

Fuente: Conicyt (2013 y 2014c)

Nota: Se entenderá por Año Concurso al año de apertura del concurso, independiente del año de evaluación, adjudicación y/o ejecución de los proyectos (Conicyt, 20I3)

Según el gráfico 6 , se puede observar el crecimiento de las posiciones postdoctorales adjudicadas en los distintos concursos Conicyt, para realizar proyectos de investigación tanto dentro como fuera de Chile, respecto al número de titulados de $\mathrm{PhD}$ en el mismo año, durante el período 2007 y 2013.

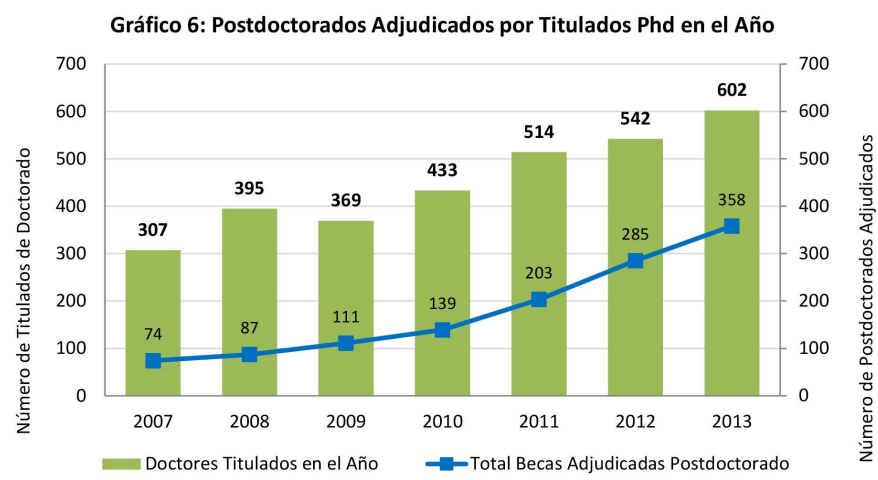

Fuente: Elaboración Propia en base a Conicyt (2014a y 20I4b), Sies (20I3) y Fondecyt (2014)
El porcentaje de postdoctorados adjudicados en el año respecto al número de titulados de doctorados en el mismo año, durante el período señalado 2007-2013, tiene sus puntos más bajos en el 2008 con $22 \%$, pero a partir de ese año comienza un crecimiento que llega a su nivel máximo una década después, cubriendo al $59 \%$ de los titulados de doctorado en ese mismo año (ver gráfico 7).

Gráfico 7: Porcentaje de Postdoctorado Adjudicados por Conicyt respecto al total de Titulados Doctorado en Chile en el mismo Año

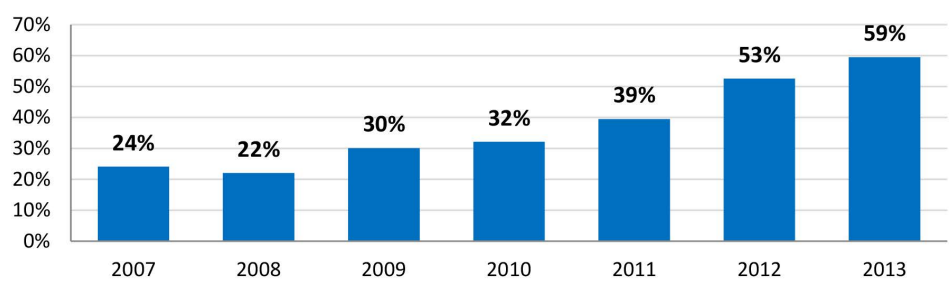

Fuente: Elaboración Propia en base a Conicyt (20I4a y 20I4b), Sies (20I3) y Fondecyt (2014)

Si comparamos el año 2013 con algunos países de referencia podemos decir que en Chile, 3 de cada 5 doctores eligen el postdoctorado como mecanismo para financiar su investigación dentro de la academia (59\%). En Finlandia por cada tres nuevos graduados de Ph.D existe sólo una posición postdoctoral disponible (32\%). Si consideramos a los países referentes del gráfico 8 , el promedio alcanza al $30 \%$ de cobertura postdoctoral.

Gráfico 8: Comparación Internacional Porcentaje de Postdoctorado adjudicados respecto al total de Titulados Doctorados en el mismo Año (2013)

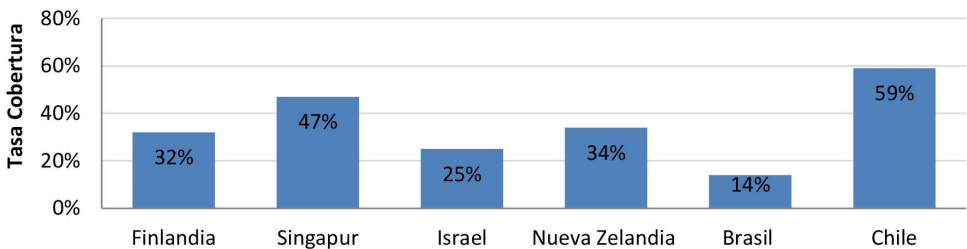

Fuente: Elaboración Propia en base a OECD (20II), Banco Mundial (2013), Statistics Finland (20I4), Education Counts (20II), Ricyt (20II), Conicyt (20I4a y 20I4c), Sies (20I3) y Fondecyt (20I4)

ISSN: 07 I8-2724. (http://www.jotmi.org) 
El alto porcentaje que Chile exhibe, demuestra que el postdoctorado es la principal vía que están tendiendo los investigadores para insertarse laboralmente en la academia. Incluso superando por amplio margen los estándares internacionales señalados.

\section{2.- Inserción en la Industria}

La industria es otra fuente de inserción laboral para los investigadores con grado de doctor. No obstante, Chile no ha logrado construir un sistema equivalente a la academia, capaz de ofrecer espacios crecientes de inserción para los investigadores en la industria. Esta situación se manifiesta en el bajo número de doctores en la industria: 185 , según la 3ra Encuesta Nacional de I+D (Minecon, 20I4). A pesar de que Chile presenta una aceptable producción de artículos científicos (Scimago, 2014), las exportaciones de bienes manufacturados de alta tecnología son casi inexistentes, como asimismo el escaso licenciamiento que realizan extranjeros por patentes y tecnologías desarrolladas por investigadores chilenos (Benavente, 2004).

Identificando este problema, Conicyt creó en 2009 el Programa de Atracción e Inserción de Capital Humano Avanzado (PAl), el cual posee una línea de inserción en la industria con la finalidad de fomentar la vinculación entre el sector productivo y la investigación científica, mediante la contratación de doctores al interior de las empresas para realizar proyectos de I+D. El instrumento financia, por un lapso de tres años, la inserción de un investigador en la empresa; mientras que la renta podrá variar en el rango entre \$18 y 27 millones de pesos anuales, donde se incluye parte importante de las remuneraciones de los investigadores al interior de la compañía (el subsidio corresponderá a un $80 \%, 50 \%$ y $30 \%$ de la renta a percibir por cada investigador durante el primer, segundo y tercer año respectivamente).
De ese modo, el programa busca estimular un cambio cultural al interior de las empresas, motivando la implementación de líneas de I+D con la incorporación de capital humano avanzado en el sector productivo (Conicyt, 2012). Los resultados del número de investigadores insertados en la industria pueden observarse en el siguiente gráfico 9 .

Gráfico 9: Inserción de investigadores en la Industria (Concurso anual Conicyt)

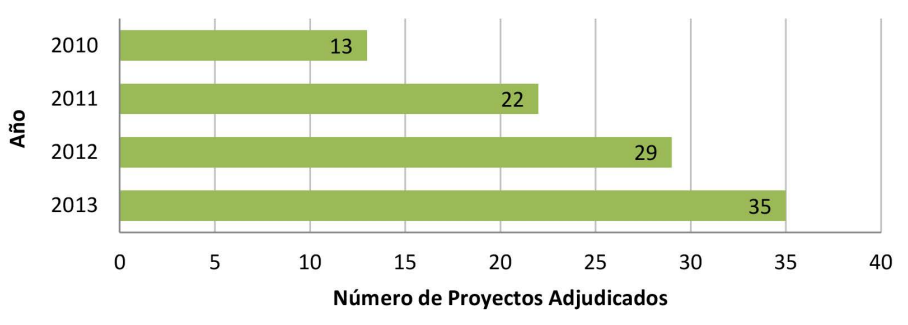

Fuente: Conicyt (20I4b)

Nota: En sus bases concursables, el instrumento de Inserción en el Sector Productivo del PAI menciona que financia "proyectos" que fomentan la vinculación entre el sector productivo y la academia, mediante la inserción de doctores en trabajos de investigación, desarrollo e innovación (I+D+i) al interior de las empresas. Por ello, en el gráfico se optó por el concepto de proyectos adjudicados para señalar la cantidad de investigadores con grado de doctor que el instrumento logró insertar en el sector productivo.

Se puede afirmar que Chile tiene una baja presencia de científicos en el mundo productivo, con cerca de 0,4 investigadores por cada mil trabajadores (Ricyt, 20I I), lo cual está lejos del promedio OECD de 7,6 (OECD, 20II). Por tanto, aquello redunda en una débil participación del mundo privado en temas de I+D, pues poco más de 200 firmas en Chile están interviniendo directamente en proyectos de innovación. Esta baja participación se explica por las condiciones de alto riesgo e incertidumbre asociados a un proyecto tecnológico, además del escaso entendimiento de la I+D por parte del sector privado e inexistencia del capital de riesgo que financie estas actividades, así la ausencia de estos factores conspira para que la industria chilena no tenga un acercamiento mayor a la ciencia de base (Benavente, 2006). 


\section{3.- Inserción a través del Emprendimiento}

En este camino de inserción es posible realizar una serie de inferencias a partir de los datos entregados en la 3era Encuesta de Gasto en I+D (Minecon, 2014). En el capítulo "Gasto I+D Empresa", se observa que las grandes empresas ejecutaron $84 \%$ (correspondiente al porcentaje del gasto total de $\$ 134$ mil millones de pesos, en 2012); mientras que las pequeñas participaron con $5 \%$, según se puede apreciar en el gráfico 10 (Minecon, 20I4).Aquello da cuenta del nivel de participación de nuevas empresas (emprendimientos) con base científica y tecnológica.

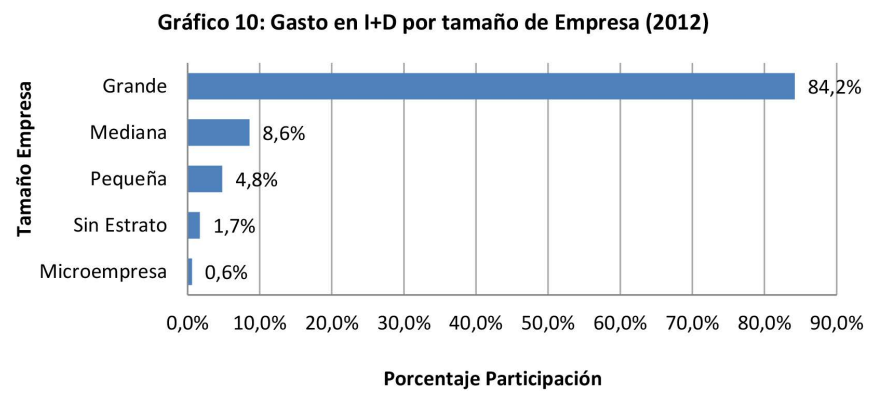

Fuente: Minecon (2014)
En tanto, el reporte "Global Entrepreneurship Monitor 2013 " señala que el $12,7 \%$ de los emprendimientos en etapas iniciales y el 3,3\% de los emprendimientos establecidos declaran que usan tecnologías (o procesos) muy nuevas en sus negocios. El mismo documento señala que, en específico, el factor de transferencia de investigación y desarrollo en el contexto nacional sigue presentando un importante déficit, el que se traspasa entre los principales actores del sistema de I+D y la manera en que puede favorecer a las nuevas empresas (Amorós y Acha, 2014).

Una línea de financiamiento que podría involucrar a nuevos doctores es el Programa de Incentivo Tributario, que gestiona y administra la Corporación de Fomento de la Producción (Corfo), que establece una rebaja en impuestos para las empresas que invierten al menos el $35 \%$ de sus recursos en actividades de I+D. Dentro de la declaración del gasto para I+D se permite la contratación de nuevo personal para labores de investigación. A fines de 2013, Corfo había certificado 184 proyectos de I+D, como se observa en el gráfico II.

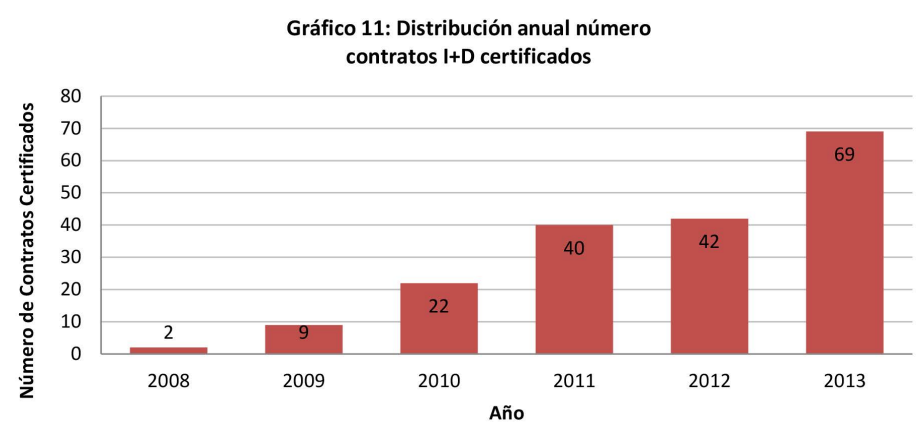

Fuente: Elaboración Propia en base a Minecon (2013)

Aunque los esfuerzos públicos para crear nuevas empresas con base científica y tecnológica son crecientes, en términos generales todavía es un formato de inserción reciente en Chile y no lo suficientemente robusto para poder extraer resultados concluyentes. 


\section{4.- Conclusiones}

Para el 2018, Chile prácticamente duplicará el número de doctores. Este es hecho muy relevante para todo el país, ya que el capital humano avanzado es fundamental para generar mayor progreso económico, social y cultural en la dinámica de las sociedades modernas. Al mismo tiempo, este incremento generará una fuerte presión en el Sistema Nacional de Innovación (SNI), sobre todo en la academia, por alcanzar una inserción laboral acorde a ese crecimiento de capital humano avanzado.

En Chile, ha existido históricamente una alta preferencia de los nuevos investigadores con grado de doctorado por realizar su carrera en el mundo académico. Este factor, entre tantos otros, explica los bajos niveles de innovación que la industria local exhibe en la actualidad. Al mismo tiempo, la empresa privada tampoco ha dado suficientes muestras de absorber y utilizar este capital humano para sus labores productivas. Para intentar cerrar esa brecha, el sector público ha realizado esfuerzos para unir la investigación científica con actividades al interior de la industria. Sin embargo, los resultados han sido discretos, debido a que no existe una política pública trasversal y a largo plazo destinada a enfrentar en profundidad la relación ciencia-empresa.

Tampoco las universidades chilenas han sido proactivas en la generación de vínculos hacia el mundo productivo. Por ejemplo, para conformar un emprendimiento de base científica es necesario que las universidades implementen políticas de propiedad intelectual dentro de sus planteles con el objeto de regular la apropiación del conocimiento científico. Pocas universidades chilenas han logrado avanzar en esta línea, con modelos que privilegian las ganancias de la universidad en detrimento de la generación de un spin off que apoye al investigador. Tampoco existe en Chile un marco legal claro que permita a los investigadores transferir hacia la industria productos y/o servicios financiados con fondos públicos.

Actualmente, el instrumento de política pública más usado por los Ph.D en Chile son las becas de posicionamiento postdoctorales que representan un puente entre la formación de doctorado y su posterior inserción laboral en el mundo académico. Desafortunadamente, será difícil sostener esta política en el tiempo, pues las universidades están alcanzando niveles de saturación respecto a la cantidad de investigadores que pueden insertar. Bajo las actuales tasas de oferta de postdoctorado, la academia chilena corre el riesgo de tener un gran número de investigadores con los requisitos necesarios para su inserción plena (doctorado en las mejores universidades del mundo y postdoctorado completo), pero probablemente las plazas permanentes de investigación no aumenten del mismo modo. Esto podría causar tensión en el sector académico chileno.
Por otro lado, se continuará generando desequilibrios en la producción y resultados de la investigación, enfocados en su gran mayoría a fortalecer el sistema académico y debilitando la eventual transferencia tecnológica hacia la sociedad. Este desbalance en la presencia de nuevos doctores en la academia respecto a la industria es un hecho preocupante, pues acota las oportunidades de desarrollo científico y tecnológico, al incentivar a que los nuevos doctores vean en las universidades como su principal polo de inserción. De esa manera, las empresas no obtienen el recurso humano para involucrarse en proyectos de investigación, evitando que actualicen su oferta tecnológica.

Según datos internacionales recogidos de países referentes en torno a la inserción de investigadores en la academia, las universidades son esencialmente un espacio de excelencia que solo inserta anualmente un tercio de la nueva generación de titulados de doctorado; el resto va a trabajar en la industria. Con esa mirada, se intenta empujar la producción académica hacia la frontera de la ciencia y, por otro lado, la industria obtiene las suficientes capacidades para absorber ese conocimiento.

Para concluir, creemos que es absolutamente necesario hacer frente al desafío de la inserción laboral de doctores en el sector industrial y fomentar los emprendimientos de base científica. Una "inflación académica" desconectada del mundo industrial y empresarial es inconsecuente con una política pro-innovación que intenta crear nuevos productos y servicios demandados por el mercado. Es absolutamente necesario que el Estado diseñe nuevos instrumentos e incentivos para que el sector privado capture y se beneficie del capital humano altamente especializado. Por otro lado, las universidades deben ser capaces de generar políticas de propiedad intelectual que permitan administrar y gestionar de mejor manera los activos que surgen de la apropiación del conocimiento científico. 


\section{5.- Apéndice}

\section{a) Consideraciones metodológicas}

La estimación de los titulados de doctorado que ingresarían al Sistema Nacional de Innovación se estimó considerando el total de estudiantes que iniciaron sus estudios de doctorado, becados por el Ministerio de Educación y abarcando cuatro instrumentos de beca de doctorado: Beca de Doctorado Nacional de Conicyt; Becas de Doctorado Internacional de Conicyt (concurso vigente hasta 2009); Becas de Doctorado en el Extranjero Becas Chile (concurso vigente desde 2009); y Beca de Doctorado en Chile para Extranjeros sin Permanencia Definitiva en Chile.

En el documento "25 Años de Becas de Doctorado" (Conicyt, 2014a) se informa el número de becarios que iniciaron estudios en estos cuatro instrumentos desde 1988, año en que solo existía la posibilidad de realizar estudios dentro de territorio nacional. Desde 1999 se incorpora el concurso de doctorado internacional, como se observa en la tabla I (ver anexo)

Para calcular la cantidad de becarios que se titularán desde 2014 al 2018, se consideró una período de éxito de 6 años, a partir desde que el beneficiario inicia sus estudios y logra titularse. Esto debido a que las bases de los mencionados concursos estipulan: "La duración de los beneficios de la beca de Doctorado, será de carácter anual, renovable hasta un máximo de cuatro (4) años contados desde el ingreso del becario al programa de estudios y sujeto al rendimiento académico obtenido por éste/a" (Conicyt, 20l4d). Al mismo tiempo, se estipula un plazo máximo de 24 meses, una vez finalizada la beca, para entregar la certificación que otorga el grado académico de Doctorado de la institución correspondiente; además, el becario puede solicitar un permiso de hasta 6 meses para suspender sus estudios, bajo una razón justificada; y también se considera la extensión de la asignación de manutención mensual para becarias con permiso de pre y post natal hasta por cuatro meses en total, durante la vigencia de la beca, donde los meses utilizados por este motivo no se considerarán para el cómputo del periodo original de la beca (Conicyt, 20l4b).
Las mismas bases indican que "CONICYT se reserva el derecho de exigir a los/as becarios/as la restitución de la totalidad de los beneficios económicos pagados en caso de que sean eliminados, suspendan o abandonen sus labores de estudios e investigación, sin causa justificada, así como a quienes no cumplan con las obligaciones inherentes a su condición de becario/a establecidas en las presentes bases 0 hayan alterado sus antecedentes o informes; además, éstos no podrán postular o participar nuevamente en cualquiera de los concursos del DSN $664 / 2008$ y sus modificaciones, del Ministerio de Educación". Dada estas sanciones, se puede suponer que la tasa de deserción es relativamente pequeña.

Considerando que los programas de Doctorado tienen una duración de 4 años, la permanencia de los estudiantes en sus programas académicos en Chile tiende a superar dicho período; de esa manera, la distancia entre los tiempos de graduación mínimo y máximo tiende a situarse en los 6 años, donde la máxima permanencia más frecuente son 8 años (Munita y Reyes, 20I2).

Con estos datos podemos establecer escenarios que permitan calcular el regreso de los titulados de doctorado (tabla 2). La mayoría de ellos cumplirá su titulación en 6 años (supuesto 60\% del total de becas otorgadas en el año t-6), otro porcentaje lo hará en 8 años ( $20 \%$ del total de becas otorgadas en el año t-8) y una porción menor lo terminará en 10 años ( $10 \%$ del total de becas otorgadas en el año $t-10)$; mientras que otro número de estudiantes nunca terminará sus estudios ( $10 \%$ del total de becas otorgadas en el año $t$ ). 


\section{Anexo Tabla I y 2}

\begin{tabular}{|l|l|l|l|l|l|l|l|l|l|l|l|l|l|l|}
\hline $\begin{array}{l}\text { Becarios CONICYT } \\
\text { por año } \\
\text { inicio Beca }\end{array}$ & 1999 & 2000 & 2001 & 2002 & 2003 & 2004 & 2005 & 2006 & 2007 & 2008 & 2009 & 2010 & 2011 & 2012 \\
\hline Becarios Nacional & 96 & 64 & 114 & 181 & 138 & 198 & 213 & 310 & 425 & 448 & 547 & 500 & 563 & 581 \\
\hline Becarios Internacional & 3 & 4 & 21 & 19 & 26 & 34 & 43 & 101 & 148 & 321 & 261 & & & \\
\hline $\begin{array}{l}\text { Becarios } \\
\text { Becas Chile }\end{array}$ & & & & & & & & & & & 344 & 346 & 500 & 392 \\
\hline $\begin{array}{l}\text { SUBTOTAL Becarios } \\
\text { Chilenos en } \\
\text { el Extranjero }\end{array}$ & 3 & 4 & 21 & 19 & 26 & 34 & 43 & 101 & 148 & 321 & 605 & 346 & 500 & 392 \\
\hline $\begin{array}{l}\text { Becarios Extranjeros } \\
\text { en Chile }\end{array}$ & & & & & & & 3 & 28 & & 64 & 35 & 73 & & \\
\hline TOTAL Becarios & 99 & 68 & 135 & 200 & 164 & 232 & 259 & 439 & 573 & 833 & 1187 & 919 & 1063 & 973 \\
\hline
\end{tabular}

Total de Doctorados - Becarios por año de inicio beca. Fuente: Conicyt (20I4a)

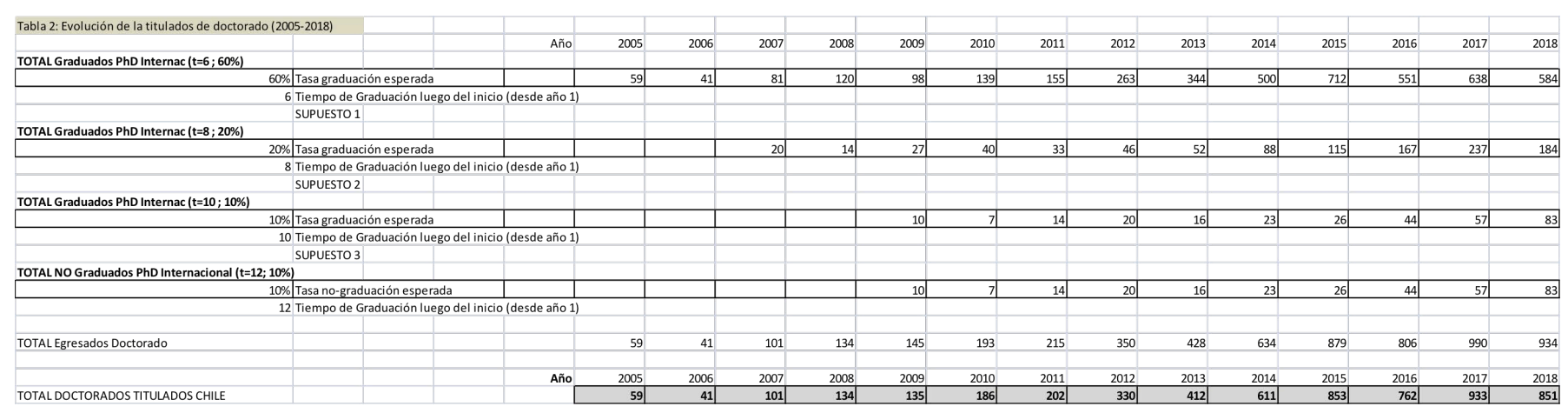

Evolución de los titulados de doctorado (2005-20I8). Fuente: Elaboración Propia en base a Conicyt (2014a)

ISSN: 07 I8-2724. (http://www.jotmi.org)

Journal of Technology Management \& Innovation (c) Universidad Alberto Hurtado, Facultad de Economía y Negocios. 


\section{Referencias}

AGUILERA, J. M. (2012). Chile's Research Planning Aims High. Science, 336 (6088), 1505-I506. doi:http://dx.doi. org//0.1 I26/science.336.6088.1505

AMORÓS, J. y Acha, A. (20|4). Global Entrepreneurship Monitor Reporte Nacional de Chile 2013. Santiago de Chile: Universidad del Desarrollo. http://negocios.udd.cl/gemchile/ files/2014/06/Reporte-GEM-Chile-2013-web.pdf

AMORÓS, J.E., Altienza, M., \& Romani, G. (2008). Formal and Informal Equity Funding in Chile. Estudios de Economía, 35(2), I79-194. doi:http://dx.doi.org/I0.4067/S07/852862008000200004

AMORÓS, J. y Echecopar, G. (2008). Emprender en Chile: ¿Estamos en el camino correcto? En P. Cortez (ed.), Emprendimiento e Innovación en Chile: Una Tarea Pendiente (pp. 8I-128). Santiago de Chile: Universidad del Desarrollo. Recuperado de http://cei.udd.cl/files/2010/I0/ Libro-Emprendimiento-e-Innovacion-en-Chile-Una-tareapendiente-Patricio-Cortes-Final.pdf

ASTUDILLO, P., Blondel, C., Norambuena,T. \& Soto, K. (2012). Chile's Research Planning Falls Short. Science, 336(6080), 412. doi:http://dx.doi.org// 0.1 | 26/science.336.6080.4 I2-a

AURIOL, L. (2010). Careers of Doctorate Holders. OECD Science, Technology and Industry Working Papers. doi:http:// dx.doi.org//0.1787/5kmh8phxvvf5-en

BANCO MUNDIAL (2013). Researchers in R\&D (per million people). Revisado en I de Octubre 20I4, de Science \& Technology Indicators. Recuperado de http://data. worldbank.org/indicator/SP.POP.SCIE.RD.P6

BENAVENTE, J. (2006). Antecedentes para el Diseño de una Política Tecnológica Nacional. Santiago de Chile: Facultad de Economía y Negocios de la Universidad de Chile. Recuperado de http://www.econ.uchile.cl/uploads/ publicacion/67/402d6-ff7f-46dc-8d4d-be53383d9c76.pdf

BENAVENTE, J. (2004). Innovación Tecnológica en Chile: Dónde Estamos y Qué se Puede Hacer. Documentos de Trabajo $n^{\circ}$ 295. Santiago de Chile: Banco Central de Chile. Recuperado de http://www.bcentral.cl/estudios/ documentos-trabajo/pdf/dtbc295.pdf

BENAVENTE, J. \& Price J. (20I4). Evolution of the Public Institutions of Science, Technology, and Innovation in Chile: 1990-2012. En: G. Crespi \& G. Dutrénit (eds.), Science, Technology and Innovation Policies for Development (pp.4562). Suiza: Springer International Publishing. doi:http://dx.doi. org//0.1007/978-3-3/9-04/08-7
BERNASCONI, A. (2005). University entrepreneurship in a developing country: The case of the P. Universidad Católica de Chile, 1985-2000. Higher Education, 50(2), 247-274. doi:http://dx.doi.org/I0.1007/s I0734-004-6353-I

BROOKS, H. (1994). The relationship between science and technology. Research Policy, 23(5), 477-486. doi:http:// dx.doi.org/10.1016/0048-7333(94)0100I-3

CATANZARO, M. (2014). Chile puts plan for science ministry on hold. Nature, 507(7493), 4/2-4I3. doi:http:// dx.doi.org//0.1038/5074I2a

CHANDRA, A., \& Medrano Silva, M. A. (20I2). Business Incubation in Chile: Development, Financing and Financial Services. Journal of Technology Management \& Innovation, 7(2), I-I3. doi:http://dx.doi.org//0.4067/s07/8272420 I 200020000 I

COCKBURN, I. M., \& Henderson, R. M. (1998). Absorptive Capacity, Coauthoring Behavior, and the Organization of Research in Drug Discovery. The Journal of Industrial Economics, 46(2), I57-182. doi:http://dx.doi. org/I0.1 III//1467-645I.00067

COHEN, W. M., Nelson, R. R., \& Walsh, J. P. (2002). Links and Impacts: The Influence of Public Research on Industrial R\&D. Management Science, 48(I), I-23. doi:http://dx.doi. org/ $/ 0.1287 / \mathrm{mnsc} .48$. I.I.14273

Comisión Nacional de Investigación Científica y Tecnológica Conicyt (20I2). Memoria de Gestión 20II. Santiago de Chile: Ministerio de Educación, Gobierno de Chile. Recuperado de http://www.conicyt.cl/documentos/ MemoriaCONICYT20II.pdf

Comisión Nacional de Investigación Científica y Tecnológica Conicyt (2013a). Ciencia y Tecnología para Chile. Santiago de Chile: Ministerio de Educación, Gobierno de Chile. Recuperado de http://www.conicyt.cl/wp-content/ uploads/2012/07/Conicyt_Brochure_2013_sp.pdf

Comisión Nacional de Investigación Científica y Tecnológica Conicyt (2013b). Compendio Estadístico Concursos de CONICYT 2008-2012. Santiago de Chile: Ministerio de Educación, Gobierno de Chile. Recuperado de http://www.conicyt.cl/wp-content/uploads/2013/07/ CompendioEstadistico2008-20I2.pdf

Comisión Nacional de Investigación Científica y Tecnológica Conicyt (2014a). 25 Años de Becas de Doctorado CONICYT. Santiago de Chile: Programa de Formación de Capital Humano Avanzado, Ministerio de Educación, Gobierno de Chile. Recuperado de http://www.conicyt. $\mathrm{cl} / \mathrm{wp}$-content/uploads/2012/07/25-Anios-Becas-deDoctorado-CONICYT.pdf 
Comisión Nacional de Investigación Científica y Tecnológica Conicyt (2014b). Memoria de Gestión (2010-2013). Santiago de Chile: Ministerio de Educación, Gobierno de Chile. Recuperado de http://www.conicyt.cl/wp-content/ uploads/20 I 2/07/MEMORIA-CONICYT-20 I0-20I3.pdf

Comisión Nacional de Investigación Científica y Tecnológica Conicyt (20I4c). Becas de Postdoctorado. Santiago de Chile: Programa de Formación de Capital Humano Avanzado, Ministerio de Educación, Gobierno de Chile. Recuperado de http://www.conicyt.cl/becas-conicyt/category/basesconcursales/becas-en-el-extranjero/becas-de-postgradobecas-chile/becas-de-postdoctorado

Comisión Nacional de Investigación Científica y Tecnológica Conicyt (20l4d). Bases Concursables. Santiago de Chile: Programa de Formación de Capital Humano Avanzado, Ministerio de Educación, Gobierno de Chile. Recuperado de http://www.conicyt.cl/becas-conicyt/category/basesconcursales

Corporación de Fomento a la Producción Corfo (2013). Memoria Corfo 2010-2013. Santiago de Chile: Ministerio de Economía, Gobierno de Chile. Recuperado de http:// repositoriodigital.corfo.cl/handle/ I I373/9877

Davis, G. (2005). Doctors Without Orders. American Scientist, 93(3 supplement). Recuperado de http://www. sigmaxi.org/docs/default-source/Programs-Documents/ Critical-Issues-in-Science/postdoc-survey/highlights

Education Counts (20II). Research Performance. Revisado en I de Octubre de 2014, de Statistics, Ministerio de Educación, Gobierno de Nueva Zelandia. Recuperado de http://www.educationcounts.govt.nz/statistics/tertiary_ education/research

FELLER, J.C. (20I I). El Sistema Chileno de Fomento a la Ciencia y Tecnología: Una Evaluación desde la Óptica de Distintos Protagonistas del Quehacer Nacional. Santiago de Chile: Sociedad Chilena de Biología. Recuperado de https:// consejosociedades.files.wordpress.com/20 I3/06/informeevaluacion-politicas-cyt-final-I.pdf

Fondo Nacional de Desarrollo Científico y Tecnológico (20/4). Concurso Postdoctorado. Santiago de Chile: Ministerio de Educación, Gobierno de Chile. Recuperado de http://www.conicyt.cl/fondecyt/category/concursos/ postdoctorado

FORAY, D., \& Lissoni, F. (2010). University Research and Public-Private Interaction. Handbook of the Economics of Innovation,Vol. I, 275-3 I4. doi:http://dx.doi.org/doi:10.1016/ s0169-7218(I0)01006-3
FOX, M. F., \& Stephan, P.E. (200I). Careers of Young Scientists: Preferences, Prospects and Realities by Gender and Field. Social Studies of Science, 31(1), 109-122. doi:http://dx.doi. org/doi: I0.1 I77/030631201031001006

FREEMAN, R. (1989). Labor Market in Action. Cambridge, MA: Harvard University Press Harvard University Press, Estados Unidos

GAGLANI, S. (2009). Investing in our Future:Ways to Attract and Keep Young People in Science and Technology. Toward an R\&D Agenda for the New Administration and Congress: Perspectives from Scientists and Economists, presentado en conferencia de National Buraeu for Economic Research, Science and Engineering Workforce Project Workshop. Cambridge, MA, Estados Unidos

GEUNA, A., \& Nesta, L. J. J. (2006). University patenting and its effects on academic research: The emerging European evidence. Research Policy, 35(6), 790-807. doi:http://dx.doi. org/doi:10.1016/j.respol.2006.04.005

GULBRANDSEN, M., \& Etzkowitz, H. (1999). Convergence between Europe and America: the transition from industrial to innovation policy. The Journal of Technology Transfer, 24(2/3), 223-233. doi:http://dx.doi.org/ doi:10.1023/a:10078595233/4

GURA, T. (2012). Training: Workshops that work. Nature, 488(74II), 4I9-420. doi:http://dx.doi.org//0.1038/nj74II $419 \mathrm{a}$

HAEUSSLER, C., \& Colyvas, J. A. (20I I). Breaking the Ivory Tower: Academic Entrepreneurship in the Life Sciences in UK and Germany. Research Policy, 40(I), 4I-54. doi:http:// dx.doi.org/I0.1016/j.respol.2010.09.012

JIMÉNEZ, A. (201I). La Difícil Ruta del Emprendimiento en la Academia. Observatorio Económico, 56. Recuperado de http://fen.uahurtado.cl/wp-content/uploads/20I I/09/Oe56web-okl.pdf

KIDD, J. M., \& Green, F. (2006). The careers of research scientists: Predictors of three dimensions of career commitment and intention to leave science. Personnel Review, 35(3), 229-25I. doi: I0.1 I08/004834806I0656676

LEHRER, M., \& Asakawa, K. (2004). Pushing Scientists into the Marketplace: Promoting Science Entrepreneurship. California Management Review, 46(3), 55-76. doi:http:// dx.doi.org/|0.2307/4| |6622| 
MARTíNEZ, R. (1998). Aproximaciones Teóricas a los Procesos de Inserción Laboral. Revista del Ministerio de Trabajo y Asuntos Sociales, 25, 65-9I. Recuperado de http:// www.mtas.es/Publica/revista/numeros/26/info3.pdf

Ministerio de Economía (2013). Ley sobre Incentivo Tributario a la I+D: principales resultados. Santiago de Chile: División de Estudios, Gobierno de Chile. Recuperado de http://www.economia.gob.cl/wp-content/uploads/2013/07/ Bolet\%C3\%ADn-Incentivo-Tributario-a-la-I+D_junio.pdf

Ministerio de Economía (20I4). Resultados 3era Encuesta Nacional sobre Gasto y Personal en Investigación y Desarrollo (I+D) 20II-20I2. Santiago de Chile: Gobierno de Chile. Recuperado de http://www.economia.gob.cl/wpcontent/uploads/2014/0 I/Presentacion-Resultados-3raEncuestas-sobre-Gasto-y-Personal-I+D.pdf

Ministerio de Educación (2010). Objetivos ¿Qué es Becas Chile? Santiago de Chile: Gobierno de Chile. Recuperado de http://www.becaschile.cl/index3.php?id_ contenido=20988\&id_portal=60\&id_seccion $=4276$

Ministerio de Educación (2013). Bases de Concurso de Propuestas de Planes de Mejoramiento Institucional para Convenios de Desempeño de Apoyo a la Innovación en Educación Superior. Santiago de Chile: Gobierno de Chile. Recuperado de http://www.mineduc.cl/usuarios/MECESUP/ File/20 I3/convocatoria20 I3/Bases_Innovacion_\%20 20I3(Res_N305de20I3).pdf

MOWERY, D. C., \& Sampat, B. N. (2004). The Bayh-Dole Act of 1980 and University? Industry Technology Transfer: A Model for Other OECD Governments? The Journal of Technology Transfer, 30(I-2), II5-I27. doi:http://dx.doi. org/I0.1007/s I096I-004-436I-z

MUNITA, M. y Reyes, J. (2012). El Sistema de Postgrado en Chile: evolución y proyecciones para las Universidades del Consejo de Rectores. Santiago de Chile: Consejo de Rectores de las Universidades Chilenas. Recuperado de http://www.consejoderectores.cl/web/pdf/Libro_sistema_ postgrado_chile_CRUCH.pdf

MURRAY, F., \& Stern, S. (2007). Do formal intellectual property rights hinder the free flow of scientific knowledge? Journal of Economic Behavior \& Organization, 63(4), 648687. doi:http://dx.doi.org//0.1016/j.jebo.2006.05.017

NEUMANN, R (2010). The Training of New Scientists. International Encyclopedia of Education, 322-327. doi:http:// dx.doi.org/I0.1016/B978-0-08-044894-7.00880-0
Organisation for Economic Co-operation and Development \& Banco Mundial (2009). Reviews of National Policies for Education:Tertiary Education in Chile. Paris Francia: OECD Publishing. Recuperado de https://files.nyu.edu/ft237/public/ OECD09_tertiary_education_chile.pdf

Organisation for Economic Co-operation and Development (20I I). OECD Science, Technology and Industry Scoreboard 201I. Paris Francia: OECD Publishing. doi:http://dx.doi. org/I0.1787/sti_scoreboard-20II-en

Official Statistics of Finland (20/4). Human resources of science and technology. Revisado en I de Octubre 2014, de Statistics Finland. Recuperado de http://www.stat.fi/til/ tthv/20I2/tthv_2012_20I4-03-20_kuv_00I_en.html

OJALA, A., \& Heikkilä, J. (2009). Entrepreneurship training for new ventures. International Entrepreneurship and Management Journal, 7(3), 297-3/0. doi:http://dx.doi. org/|0.1007/s | |365-009-0|26-3

PÉREZ, C. (2013). Pensando Chile 2050 desde los Investigadores en Posgrado. En L.S. Cabrera (ed.), Institucionalidad para el Desarrollo de Ciencia y Tecnología (pp. 83-90). Concepción Chile: Universidad de Concepción, Centro de Óptica y Fotónica. Recuperado de http://cyt2013. $\mathrm{cl} / \mathrm{wp}$-content/uploads/2013/08/libro_encuentrol.pdf

POWELL, W. W., Owen-Smith, J., \& Colyvas, J. A. (2007). Innovaton and Emulation: Lessons from American Universities in Selling Private Rights to Public Knowledge. Minerva, 45(2), |2I-I42. http://dx.doi.org//0.1007/s I I024007-9034-2

Red de Indicadores de Ciencia y Tecnología Iberoamericana e Interamericana RICYT (20II). Investigadores por sector de empleo (PF). Revisado en I de Octubre 20I4, de Investigadores por sector de empleo. Recuperado de http://db.ricyt.org/query/AR,BO,BR,CL,CO,CR,EC,ES, GT, HN,MX,PA,PE,PR,PT,PY,SV,TT,UY,VE,AL,IB/ I 990\%2C20 I I/ INVESTPFSEPER

ROACH, M. (2009). When Do Firms Use Public Research? Empirical Evidence of Knowledge Flows from Universities and Government Labs to Industrial R\&D. Working Paper, presentado en conferencia Copenhagen Bussiness School, Copenhague, Dinamarca. Recuperado de http://www2.druid. $\mathrm{dk} /$ conferences/viewpaper.php?id=5988\&cf $=32$

ROACH, M., \& Sauermann, H. (20I0). A taste for science? $\mathrm{PhD}$ scientists' academic orientation and self-selection into research careers in industry. Research Policy, 39(3), 422-434. doi:http://dx.doi.org//0.1016/j.respol.2010.01.004 
RODRÍGUEZ, E. (2004). Políticas y estrategias de inserción laboral y empresarial de jóvenes en América Latina: el desafío de la empleabilidad. Revista Latinoamericana de Ciencias Sociales, Niñez y Juventud , 2(I), 75-126. Recuperado de http://www.scielo.org.co/scielo.php?script=sci_ arttext\&pid=S|692-7| $5 \times 2004000|00004 \&| n g=e n \& t \operatorname{lng}=e s$

ROSENBERG, N. (1985). The Commercial Exploitation of Science by American Industry. En K. Clark, R. Hayes y C. Lorenz (eds), The Uneasy Alliance: Managing The Productivity-Technology Dilemma (pp. 14-5I. Cambridge: MA, Harvard Business School Press, Estados Unidos

SAUERMANN, H. y Cohen, W. (2008). What makes them tick? Employee motives and industrial innovation. NBER Working Paper no. 14443. Cambridge: MA, National Bureau of Economic Research. Recuperado de http://www.nber.org/ papers/w I4443.pdf

SAUERMANN, H. y Stephan, P. (2009). Twins or Strangers? Differences and similarities between academic and industrial science. Working Paper No. I6I I 3 Cambridge: MA, National Bureau of Economic Research. Recuperado de http://www. nber.org/papers/w16 I 3.pdf

SCIMAGO (20|4). Principales indicadores cienciométricos de la actividad científica chilena 2012. Madrid España: SClmago Research Group,Altazor Ediciones. Recuperado de http://www.conicyt.cl/informacioncientifica/files/2014/09/ Indicadores-Cienciom\%C3\%A9tricos-20I4.pdf

Servicio de Información de Educación Superior (2013). Compendio Histórico de Educación Superior. Revisado en I de Octubre de 2014, de Base Titulados Histórica 2007 a 2012 y Base Titulados 20I3. Santiago de Chile: Ministerio de Educación, Gobierno de Chile. Recuperado de http://www. mifuturo.cl/index.php/bases-de-datos/titulados

SORENSON, O., \& Fleming, L. (2004). Science and the diffusion of knowledge. Research Policy, 33(10), 1615-1634. doi:http://dx.doi.org/10.1016/j.respol.2004.09.008

STEPHAN, P. (2006). Wrapping it up in a Person:The mobility patterns of new PhDs. En A. Jaffe et al (eds) Innovation Policy and the Economy, (pp. 7I-98). Cambridge: MA, MIT Press, Estados Unidos. Recuperado de http://www.nber.org/ chapters/c0034.pdf

STEPHAN, P. (20/2). How Economics Shapes Science. Cambridge: MA, Harvard University Press, Estados Unidos. doi:http://dx.doi.org//0.4159/harvard.9780674062757
STERN, S. (2004). Do Scientists Pay to Be Scientists? Management Science, 50(6), 835-853. doi:http://dx.doi. org/ I0.1287/mnsc. I040.024 I

STEVENS, A. J. (2004). The Enactment of Bayh-Dole. The Journal of Technology Transfer, 29(I), 93-99. doi:http:// dx.doi.org/ I0.1023/b:jott.00000 I I 83.40867.52

VOGEL, G. (1999).A Day in the Life of a Topflight Lab. Science, 285(5433), I53I-1532. doi:http://dx.doi.org/I0.1 I26/ science.285.5433.1531 